\title{
Analysis of the "Get+V-ed" Structure under the Force-Dynamic Pattern
}

\author{
Liu Yang
}

School of Foreign Languages, Jilin Agricultural University, Changchun, China, 130118, China

Keywords: Force-dynamic pattern; "Get+V-ed"; Reflexive.

\begin{abstract}
The force-dynamic pattern was first put forward by cognitive linguist Talmy. He applied the physical concept to the cognitive semantics field to represent how entities interact with each other. The present article finds this theory make powerful illustration in understanding the structure of "Get+V-ed”, especially the semantic difference between different "Get+V-ed” structures.
\end{abstract}

\section{Introduction}

The structure of "Get+past participle” (referred as Get+V-ed in the present article) has long drawn a lot argumentation among different schools of the linguists. Especially about the semantic difference between "Get+past participle" and Get+reflexive+past participle (referred as Get+refl.+ V-ed in the present article), the research result has been largely diversified. The present article aims to analysis the structure under the theory of Dynamic Force, trying to find the hidden meaning and causative reasons of this structure.

Through observation and research, Talmy (2000) and Lampert (2011) have found that many structures have fully shown "Force Dynamics", and have depicted the physical form of the force dynamics. Talmy put most emphasis on analyzing the structure of "KEEP+V-ING", in the effort to illustrate how Force Dynamics function in different sentences. The raising of this theory has enlightened many related research.

\section{The Analysing Mould of Force Dynamics}

The Dynamic Force has influenced many aspects of language. It provides a totally new angle to illustrate the auxiliary verbs, and at the same time it also has affected the discourse analysis, lexical semantics and morphology syntax. It's a big trait of the analysis of the relationship between forces in Talmy's cognitive linguistics. It belong to the semantic sphere, depicting the interaction routes between the forces, including the exerting and antagonist forces. The theory can illustrate concepts like push, pull and drag, and depict some abstract concepts of psychological forces, like want, oblige and etc.

Compared to the concept of "causative" in traditional linguistics, the Force Dynamic s provides a more scientific and precise analyzing method. In discussing the terms in Force Dynamics, we are to read the next two examples:

(1a) The door is closed.

(1b) The door cannot open.

The two sentences share the same meaning, i.e. the door is closed. In (1a), the Force Dynamic doesn't appear, because there are not the pair of forces exerted on each other, and the sentence only shows a steady state; in (1b), the Force Dynamic works, some kind of force prevents the door from being opened.(The door may be stuck or locked.)

In the discourse where Force Dynamic works, there are usually two interacting forces. Because they two forces play contrary roles, one can be foregrounded, being the attention focus, which is called Agonist; the other Antagonist. In (1b), the door is the Agonist, and the force preventing it is the antagonist.

In Talmy's system of diagramming, the Agonist (Ago) will be indicated by a circle and the antagonist (Ant) by a concave figure, as shown in (2a). 

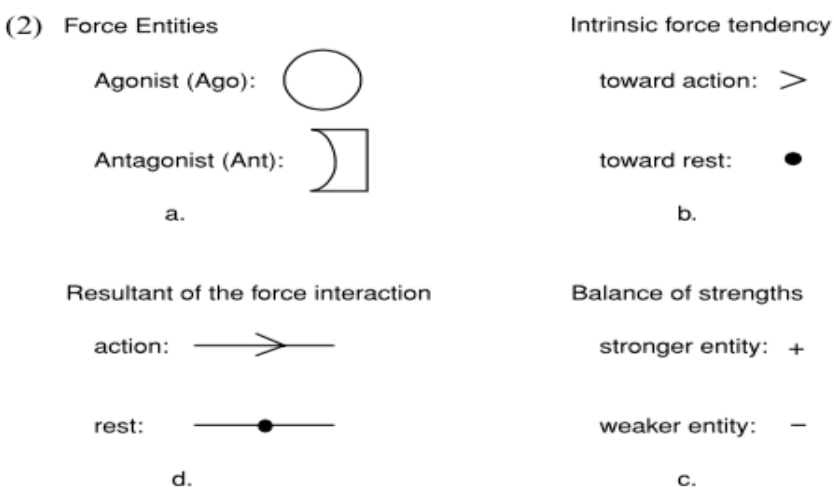

With these basic distinctions, we are to see the four most basic force-dynamic patterns, those involving steady-state opposition, as exemplified in (3). (Talmy, 2000:415)

(3) The basic steady-state force-dynamic patterns

a.

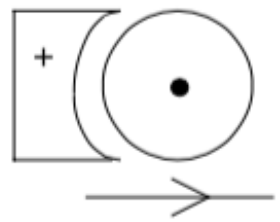

c.

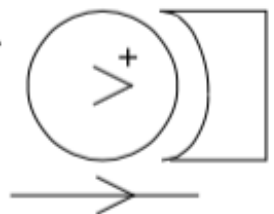

b.

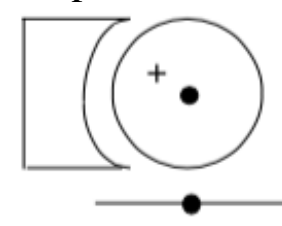

d.

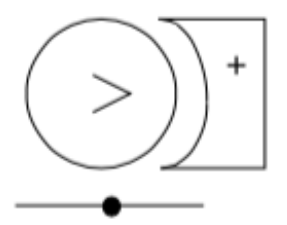

Ago's tendency $(a, b)$ : toward rest (c,d): toward action

Ago's resultant $(\mathrm{a}, \mathrm{c})$ : action $(\mathrm{b}, \mathrm{d})$ : rest

Ago's force relative to Ant's:

$(a, d)$ : lesser

(b,c): greater

In the diagram above, every pair has something in common. In the top pair of (a, b), the antagonists' tendency is toward rest, while in the bottom pair of (c, d), they are toward action. In the left column, (a,c), the resultant of the force opposition is action, while in the right column, (b, d), it is rest. More significantly, the diagonal starting at top left, (a, d), which represents the cases where the Antagonist is stronger, capture the factor of extended causation. And the diagonal starting at the top right, (b, c), which gives the cases where the Agonist is stronger, captures the "despite" factor. In fact the very concept "despite/although" can be characterized in terms of the common factor in this subset of force-dynamic patterns.

What should be put out here is the distinction of different aspects of the "forces" in force-dynamic patterns. The force can not only be referred as the concrete force exerted by some entities, but also as some abstract forces as in psychological and social fields.

1) "Physical Force" refers to the force produced by some physical entities. e.g.:

(4a) The ball was rolling along the green.

(4b)The ball kept on rolling along the green.

In (4a), there is not a force-dynamic pattern, but in (4b), the use of the verb "keep" represents this pattern. It can be seen that, in the semantic sense, there is a force keeping the ball rolling.

2) "Psychological Force" refers to the shapeless "force" created by mind or emotion. e.g.:

(5a) He didn’t close the door.

(5b) He refrained from closing the door.

In (5a) and (5b), the situation remains the same, i.e. the door was not closed. In (5a), there was not a force-dynamic pattern; but in (5b), it shows the mind conflict, which is the result between the "force" he wants to close the door and the "force" he refrains.

3) "Social Force" refers to as the motivation or refraining "force" composed of the social responsibilities and obligations. e.g.:

(6a) She's got to go to the park.

(6b) She gets to go to the park. 
In these two sentences, the force-dynamic patterns are different, but they show the same results. In (6a), she wishes to not to go to the park, but pressed by the outer force, she has to go; in (6b), she wishes to go to the park and not prevented by the outer circumstances, so goes she.

\section{Analysis of the “Get+V-ed" Structure}

Many linguists have different ideas about the semantic illustration of the "Get+V-ed" structure. In researching the force-dynamic pattern, I have found the illustrating power in understanding this structure better. We will see this more clearly by analyzing some sentences.

(7a) John got killed in an accident.

(7b) The book got torn on purpose.

In (7a), we can see that John was killed in an accident. The agent didn't appear so the responsibility has not been made clear. So in this sentence, John was the antagonist, and the verb "got" implies there is a force working, but the force the agonist exerting appears very weak. Yet it still produces serious results (John got killed). The following diagram (8) shows the situation:

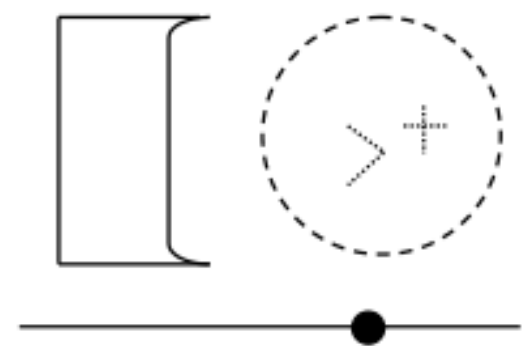

We can understand the pattern better based on the diagram. The real Agonist does not appear, its force strengthening. The Antagonist has been exerted force, so its tendency is still toward steady. This can be regarded as the first and most frequently used situation that the structure is used. In most of the "Get+V-ed" structures, the Agonists do not appear and the adverbials cannot be found there.

(7b) represents another situation: there is an adverbial in the sentence. In this case, it is a prepositional phrase. Modified by a phrase, though the Agonist is still missing, its force appears to be more strengthened. The reason for this is though the Agonist does not appear in the sentence; it does exert a force on the Antagonist. It is invisible, but not inexistent. So we can know that the use of adverbials can strengthen the force-dynamic pattern.

Another pattern for this structure is "Get+ reflexive+ past participle". Let see an example:

(9a) First, she had got herself pregnant (that was how she put it) and married the father.

(9b) You can try, but it's going to be hard. "But I got elected to the House of Representatives in 1994 because I did something daring...”

The structure of "Get + refl. +V-ed" comes from "Get +V-ed", but they differ in both semantic and pragmatic sense. The meaning of "Get $+\mathrm{V}$-ed" is to cause or make efforts, so only animate words can be used as subjects in the structure of "Get + refl. +V-ed". This structure shows the subject takes some responsibility in causing the effect, so it plays double roles, being the Antagonist and (part of) the Agonist. As in (9a), "she” cannot get pregnant by herself, but the use of "herself” here implies her will plays an important role in this action. Or she herself should be totally responsible for this. Compare with (9a), the structure of "Get +V-ed" in (9b) clearly does not bear such effects. This is better shown in the following example:

(10) She didn't try to debate the moral issues of prostitution with San-de; she was worried about her daughter's survival. 'I said, 'Sand-e, you could get yourself killed doing this,' and she said, 'Oh Mom, I'm not going to get killed.' She didn't want to hear about it or talk about it because she knew I was so scared."

In (10), the mother use the structure of "Get + refl. +V-ed" to emphasize the daughter's responsibility, implying her prostitution will lead her to death. But the daughter use the structure of "Get +V-ed" to reduce her responsibility in the potent crime.

Under the theoretical frame of Talmy's force-dynamic pattern, we can see that in the structure of "Get +V-ed", the Agonist is missing, but the force still works here. Yet in the structure of "Get + refl. 
+V-ed", the Agonist remains missing, but the appearance of the reflexive dilutes the Agonist's force. The reflexive shares some responsibility with the Agonist, or to some extent the reflexive should take all of the responsibilities, at least, subjectively. In this case, the subject and the reflexive can be regarded as the Antagonist and the Agonist. They are different words, but the same entity.

\section{Conclusion}

Talmy’s force-dynamic pattern can illustrate many "causative” structure in traditional grammar, but more precisely and intriguingly. The semantic analysis of the structure of "Get +V-ed" is better performed in the force-dynamic pattern. It can be used in explaining more structures, which are to be researched in future.

\section{Acknowledgments}

The present article has been sponsored by the Youths' Foundation in Jilin Agricultural University. The present research has been the author's related achievement.

\section{References}

[1] Langacker,R.W.Investigations in Cognitive Grammar.Berlin \& New York: Mouton de Gruyter, 2009.

[2] Lampert,M. Linking up Three Schematic Systems. The 11th International Cognitive Linguistics Conference, 2011.

[3] Talmy ,L.Toward a Cognitive Semantics. Cambridge,MA: MIT Press,2000. 